\title{
Planktonuntersuchungen in einem holländischen Meeresarm vor und nach der Abdeichung
}

\author{
CORNELIS BAKKER \\ Hydrobiologisch Instituut, Yerseke, Niederlande \\ Afd. Delta-Onderzoek
}

\begin{abstract}
Plankton investigations in a Dutch sea-arm before and after dike construction. The Veersche Gat and the Zandkreek formed a sea-arm, which since its closure has been known as the Veerse Meer. Changes in the environment and plankton before and after the closure are reviewed. For the quantitative plankton investigations the method of LoHMANN (1908) was applied. As parts of the Oosterschelde system the Veersche Gat and the Zandkreek were poly-euhaline biotopes. Partial isolation of the central stations of the Zandkreek was caused by the existence of a tidal divide at Wolfaartsdijk (Fig. 2). The effect of this divide was illustrated by aberrant chlorinity values and by different qualitative and quantitative composition of the plankton communities. The Zandkreek plankton appeared to be a neritic biocoenose, consisting of a phytoplankton community of mainly pelagic diatoms together with a zooplankton community with strongly littoral characteristics. The size of the standing crops generally increased towards the sea. Phytoplankton at the tidal divide sometimes showed an autochthonous development. Sometimes phytoplankton organisms occurring throughout the whole water body were seen to bloom notably within the area of the tidal divide. In summer a strong autochthonous development of littoral zooplankton made the standing crops within the Zandkreek larger than those of the Veersche Gat. In the second and third years after the formation of the Veerse Meer, a decline in salinity transferred this water body from a poly euhaline into a meso-polyhaline environment. This decrease in salinity was coupled with a strong eutrophication, evidenced, for example, by high phosphorous contents of the water and catsed by the discharge of water from the neighbouring polders and by pollution from sewage. The elimination of many polyhaline-marine species was associated with the gradual appearance of many brackish-water species with flagellates and dinoflagellates as the most important groups. At the same time the standing crops increased greatly. The severe winter at the beginning of 1963 resulted in a marked salinity stratification. In the course of the spring when the ice cover disappeared and the temperature of the upper layers rose, the halocline was combined with a thermocline, and this double stratification was observed until August, 1963 , when the wind gradually succeeded in mixing all layers of the water.
\end{abstract}

\section{EINLEITUNG}

In einem kleinen Meeresarm des holländischen Deltagebietes wurden Planktonuntersuchungen durchgeführt, und zwar zuerst im Jahre 1959. In dieser Zeit befand sich der Meeresarm noch in seinem ursprünglichen Zustand als marines Gezeitengebiet (Veersche Gat-Zandkreek). Die späteren Untersuchungen erfolgten nach der Abdeichung, welche aus dem Untersuchungsgebiet ein brackiges Binnengewässer (Veerse Meer) entstehen ließ. 
Die Topographie des holländischen Deltagebietes ist in Abbildung 1 schematisch dargestellt. Wir können drei Systeme unterscheiden: a) das Schelde-Aestuar mit der Westerschelde; b) die Oosterschelde, ein Meeresarm, der nicht als Flußmündung funk-

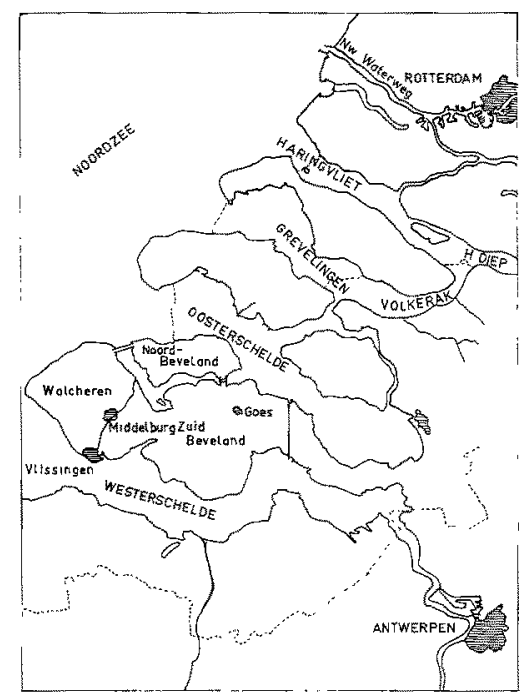

Abb. 1: Topographie des holländischen Deltagebietes. Zwischen den Inseln Zuid-Beveland, Walcheren und Noord-Beveland befand sich der ehemalige Meeresarm Veersche Gat-Zandkreek, jetzt Veerse Meer. Die fertigen Dämme sind normal eingezeichnet, die künftigen Deiche mit punktierten Linien wiedergegeben

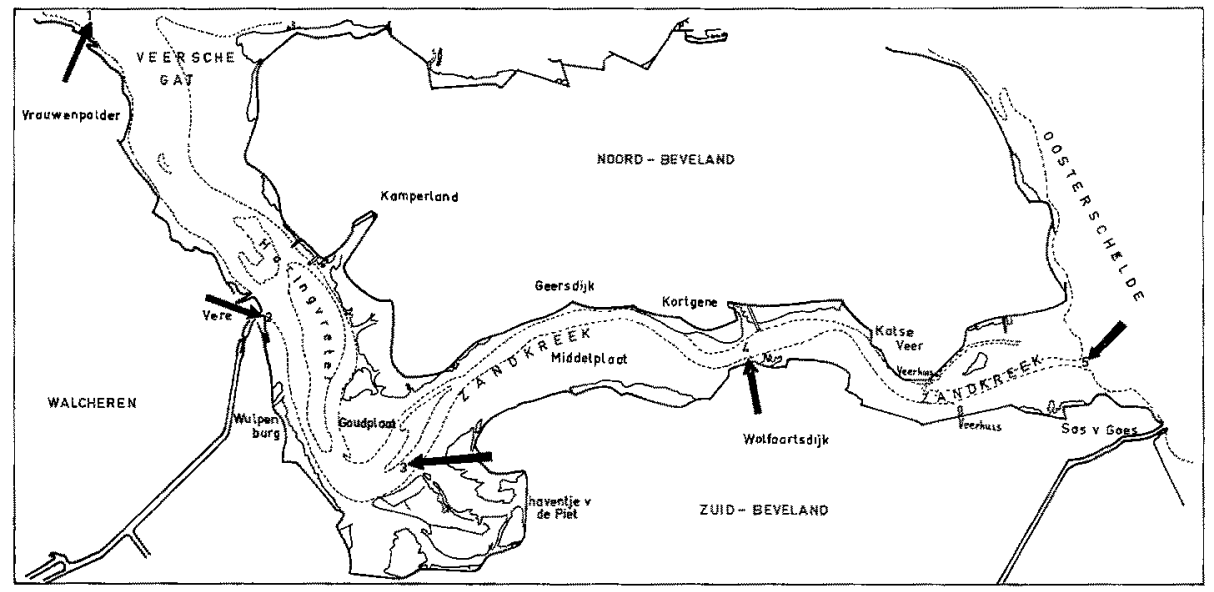

Abb. 2: Topographie des Untersuchungsgebietes: Veersche Gat-Zandkreek. Die unterbrochene Linie trennt die Fahrrinne von den bei Niedrigwasser trockenfallenden Gründen (z. B. Haringvreter, Goudplaat)

tioniert; c) das Rijn-Maas-Aestuar mit Volkerak/Grevelingen, Hollandsch Diep/Haringvliet und Nieuwe Waterweg. Veersche Gat und Zandkreek bildeten einen Teil des Oosterscheldesystems und konnten als euhaline Biotope charakterisiert werden. Der 
östliche Damm (mit Schleusen) wurde Mai 1960 fertig, der westliche (ohne Schleusen) April 1961. Für Angaben in bezug auf die Gründe der niederländischen Regierung, dem Deltadienst des Rijkswaterstaats aufzutragen, die Meeresarme abzuschließen, wird verwiesen auf VAAS (1961). Eine Einteilung des Deltagebietes auf Grund faunistischer Angaben erwähnt DEN HARTOG (1961). Eine kurze Übersicht der Kieselalgenflora der ehemaligen Zandkreek wird von Schuurmans Stekhoven (1961) gegeben. Eine Darstellung der Umwelt und des Planktons des Veersche Gat, der Zandkreek und des Veerse Meer ist in Vorbereitung (BAKKER, unpubliziert).

\section{UNTERSUCHUNGEN IM URSPRUNGLICHEN MEERESARM, VEERSCHE GAT-ZANDKREEK, IM JAHRE 1959}

\section{Beschreibung des Untersuchungsgebietes}

Abbildung 2 zeigt eine Obersicht des Gebietes mit den bedeutendsten. Fahrrinnen und bei Niedrigwasser trockenliegenden Gründen. Das Veersche Gat erstreckte sich von der Nordseeküste (Strand von Vrouwenpolder) bis Veere und die Zandkreek von Veere $b$ is an die Oosterschelde.

Im nachfolgenden Text werden Veersche Gat als VG, Zandkreek als $Z K$ und Oosterschelde als OS abgekürzt. Die Gesamtlänge der Strecke VG-ZK beträgt ungefähr $28 \mathrm{~km}$. Die Tiefe der Fahrrinne wechselt ziemlich stark: die mittlere Tiefe beträgt etwa $10 \mathrm{~m}$, doch gelegentlich werden Tiefen bis zu $17 \mathrm{~m}$, ja bis zu $25 \mathrm{~m}$ gemessen. In der ZK befand sich nahe Wolfaartsdijk ein "wantij" (English: tidal divide'): ein Wassergebiet, welches im wesentlichen nur eine vertikale Wasserbewegung aufweist, weil sich hier zwei Flutströme entgegengesetzter Richtung begegnen. Die Strömungsgeschwindigkeiten in einer "wantij" sind immer sehr gering: an der Stelle, wo die Flutwasser des VG und der OS aufeinanderstoßen bis etwa $20 \mathrm{~cm} / \mathrm{sek}$. Die höchste Strömungsgeschwindigkeit im VG betrug etwa $1,50 \mathrm{~m} / \mathrm{sek}$; der Tidenhub belief sich auf etwa $3 \mathrm{~m}$ und nahm in Richtung "wantij" infolge der Stauung geringfügig zu.

Auf 5 Stationen wurden monatlich Wasserproben genommen. Diese Stationen sind in Abbildung 2 angegeben. Station 1 liegt am Nordsee-Eingang des VG, Station 2 am VG-Eingang der ZK, Station 5 am OS-Eingang der ZK; die Stationen 3 und 4 sind die zentralen ZK-Stationen. Es wurde stets dafür gesorgt, daß die Stationen 1 und 2 um die Zeit des Hochwassers erreicht wurden, so daß die Probenentnahme an den verschiedenen Stationen immer in ungefähr derselben Phase einer Gezeitenperiode stattfand.

\section{Umwelifaktoren}

Chlorinität. Im Verlaufe eines Jahres schwankte der Chloridgehalt des Oberfä̈chenwassers zwischen 15 und $18 \%$ Cl. Die Chloridwerte für die Zeit von März 1959

Nach mündlichen Mitteilungen von Prof. Dr. K. KaLle und Dr. E. Gordecke gibt es keine gute deutsche Übersetzung dieses Begriffs; daher benutze ich hier und im folgenden den holländischen Ausdrudk ,wantij“. 
bis März 1960 sind Abbildung 3 zu entnehmen. Auf jeder Station fand eine doppelte Probenentnahme statt, das erste Mal vom Flutwasser, das zweite Mal vom Ebbewasser. Die Zahlen sind ohne Durchschnittswerte angegeben, so daß die Chlorinitätskurven eine zusammenhängende Flut- und Ebbereihe aufweisen. Es zeigt sich, daß im Winter und Frühling eine allmähliche relative Steigerung des Chloridgehalts in Richtung Ost-West auftrat. Im Sommer wiesen die zentralen ZK-Stationen 3 und 4 die relativ

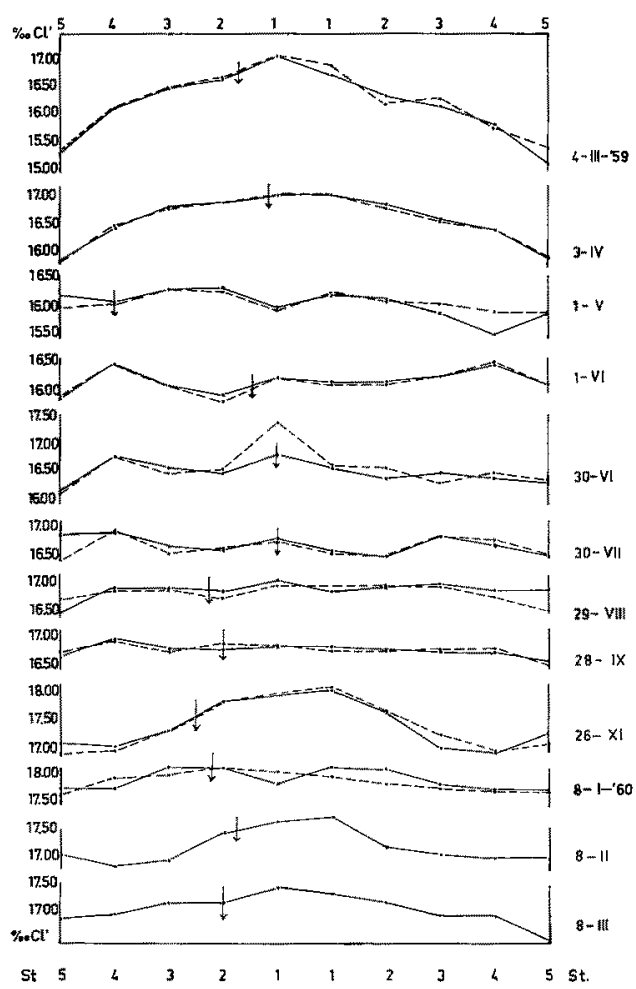

Abb. 3: Chlorinität des Oberflächen- bzw. Tiefenwassers des Veersche Gat und der Zandkreek, mit kontinuierlichen bzw. unterbrochenen Linien wiedergegeben (März 1959 bis März 1960). Die Zahlen 5-1 und 1-5 unten und oben stellen die Stationen der Probenentnahme dar (vgl. Fig. 2). Die Pfeile markieren den Zeitpunkt des Hochwassers

höchste Chlorinität auf. Diese Tatsache war zum Teil die Folge der partiellen Isolation der zentralen ZK durch die "wantij" vom Wolfaartsdijk. Dadurch wurde der Niederschlag - zusammen mit dem Polderwasser - ein sehr bedeutender Faktor für die lokale Herabsetzung des Chloridgehalts während der nassen Jahreszeiten. Ahnlich war die Steigerung der Chlorinität der zentralen ZK-Stationen, insbesondere an der Station 4, die Folge einer intensiven Verdunstung während des warmen und trockenen Sommers 1959. Chlorinitätsdifferenzen zwischen Oberflächenwasser und Tiefenwasser (10-15 m) waren infolge der starken Mischungsvorgänge durch die Gezeitenströmungen immer gering. Stabile Schichtungen konnten daher im Untersuchungsgebiet niemals entstehen, ebensowenig wie in der ganzen südlichen Nordsee. 
Temperatur. Die Temperaturdaten schließen gut an Vorstehendes an: das Wasser der ZK zeigte im Vergleich mit dem Wasser des VG eine stärkere Erwärmung während des Sommers und eine stärkere Abkühlung im Winter. Die ZK bekam so einigermaßen den Charakter eines Binnengewässers. Die Sommertemperaturen stiegen bis $20^{\circ} \mathrm{C}$, die Wintertemperaturen sanken (in Wintern ohne lang anhaltende Frostperioden) bis zu $2^{0}$ oder $3^{\circ} \mathrm{C}$ ab.

Phosphor. Die Menge des Gesamtphosphors wechselte zwischen 1 und $5 \mu$ gr. at. P/l mit Minimalwerten im Frïhling und Sommer (Hoekman 1962).

Sauerst offgehalt. Als Sättigungswert gemessen, betrug der Sauerstoffgehalt in dem turbulent strömenden Wasser meistens etwa $100 \%$ und konnte während der Entwicklungsmaxima der Phytoplanktonorganismen bis auf $150 \%$ ansteigen.

\section{Planktonuntersuchungen}

Für die quantitativen Untersuchungen wurde folgende Methodik angewendet. Zählungen wurden mit dem Reichert-Universal-Kamera-Mikroskop Me \& F ausgeführt. Die Ermittlung der Organismenzahlen pro Liter oder pro Kubikmeter erfolgte nach der Methode von LommanN (1908): Von jeder in quantitativer Hinsicht wichtigen Art wurde das Volumen bestimmt und die Anzahl der Organismen pro Volumeneinheit zum Populationsvolumen umgerechnet. Um die ganze planktonische Lebensgemeinschaft zu erfassen, wurden die Populationsvolumen aller untersuchten Arten zusammengezählt. Auf diese Weise erhält man das Gesamtplanktonvolumen pro Raumeinheit. Für die meisten Planktonorganismen gilt dieses Planktonvolumen als Maßstab der jeweiligen örtlichen Biomasse. Für Diatomeen muß ein Korrektionsfaktor wegen der manchmal sehr großen Vakuolensaftmengen einkalkuliert werden.

Das Phytoplankton. Insgesamt wurden 50 Diatomeenarten nachgewiesen, hauptsächlich pelagische Vertreter, wenige hemipelagische (Actinoptychus ssp., Actinocyclus Ebrenbergii, Aulacodiscus argus, Biddulphia aurita u. a.). Rein littorale Diatomeen, wie etwa die meisten Pennatae, sind qualitativ meistens außer Betracht gelassen worden. Die Planktongemeinschatten weisen einen stark neritischen Charakter auf. Von den Dinoflagellaten wurden etwa 25 Arten festgestellt. Temporär hohe Abundanzen wurden bei der bekannten Chrysophyceengattung Phaeocystis beobachtet. Die quantitativen Fluktuationen der Phytoplanktonpopulationen wurden im Frühling und im Sommer 1959 registriert und in Abbildung 4 dargestellt. Die Maximalwerte liegen im VG (Station 1 und 2), und zwar in der Größenordnung von 1500-2000 $\mathrm{mm}^{3} /$ $\mathrm{m}^{3}$. Station 5 zeigt ein ausgesprochenes Frühlingsmaximum. Die zentralen ZK-Stationen 3 und 4 weisen im Frühling und im Vorsommer geringere (mitunter bedeutend geringere) Volumenwerte als die VG-Stationen auf. Das kräftige Entwicklungsmaximum der OS wird durch die Frühlingsdiatomee Porosira glacialis verursacht. Im VG wird das Frühlingsmaximum gleichzeitig durch Sceletonema costatum gebildet (Abb.5). Die auffällig starke Entwicklung des Phytoplanktons im Vorsommer ist fast ausschließlich auf die Kieselalge Guinardia flaccida zurückzuführen. Die Phytoplanktonvolumina vom 30. Juli bestehen zum größten Teil aus den Peridineen Ceratium fusus und Peridinium leonis. Im Spätsommer sinkt das Gesamtphytoplanktonvolumen. Aber 
mehrere Diatomeen-Arten existieren in großer Menge, z. B. Chaetoceros radians und Biddulpbia sinensis. Die graphischen Darstellungen in Abbildung 4 zeigen, daß die Phytoplanktondichte von West nach Ost allmählich bis zu einem Minimalwert bei der Station 4, der "wantij"-Station, absinkt. Zwischen dieser Station und der OS gibt es sehr große Unterschiede. Die ZK wird also kaum durch die OS beeinflußt, und das

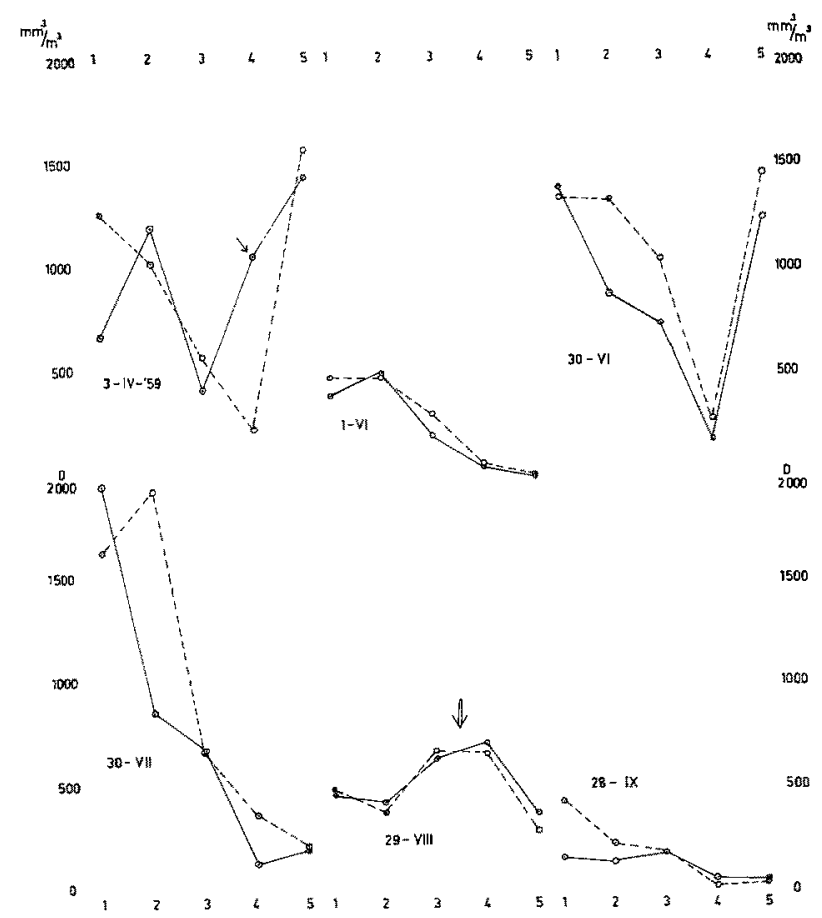

Abb. 4: Gesamtphytoplanktonvolumina $\left(\mathrm{mm}^{3} / \mathrm{m}^{3}\right)$ im Frühling und im Sommer 1959 des Veersche Gat- und Zandkreek-Flutwassers. Kontinuierliche Linien: Oberflächenwasser, unterbrochene Linien: Tiefenwasser

Flutwasser aus dem VG bestimmt die Mengenverteilung des Planktons bis an die „wantij“. Die kräftige Wirkung der Gezeiten verringert anfangs die Möglichkeiten für die Bildung eigener ZK-Populationen (Frühling und Vorsommer). Erst Ende August (Abb. 4) findet man bei den zentralen ZK-Stationen die höchsten Volumenwerte, weil jetzt einige Arten (Chaetoceros radians, Biddulphia sinensis, Rbizosolenia stolterfotbii) hier ihre Entwicklungsmaxima aufweisen.

Das große Phytoplanktonvolumen der Station 4 am 3. April 1959 (Abb. 4) kam durch die große Abundanz der littoralen Kieselalge Nitzscbia commutata zustande. Dieses Maximum war einer rein autochthonen Entwicklung zu verdanken, welche

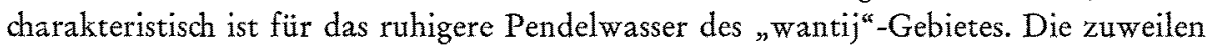
stark erhöhten Werte des Phytoplanktonvolumens des Tiefenwassers (Abb. 4) wurden

2 Die Bestimmung dieser Diatomee verdanken wir Herm A. VAN DER WERF vom Palaeobot. Lab., Geolog. Dienst, Haarlem. 
durch Sedimentation bei abnehmenden Strömungsgeschwindigkeiten verursacht. Daher sind es die Stationen 1 und 2, die ja meistens zur Hochwasserzeit geprüft wurden (Pfeile in Abb. 3), welche die größten Differenzen zwischen Oberfläche und Tiefe aufweisen.

Das August-Maximum innerhalb der $\mathrm{ZK}$ ist wahrscheinlich eine im allgemeinen nebensächliche Erscheinung, aber bezeichnend für den Zyklus des Jahres 1959 besonders im Hinblick auf den warmen Sommer. Leider war dieser Zyklus der einzige, der vor der Abdeichung studiert werden konnte, so daß keine Vergleiche mit vorhergehenden, normaleren Jahren möglich sind. Aus dem gleichen Grunde ist es nicht

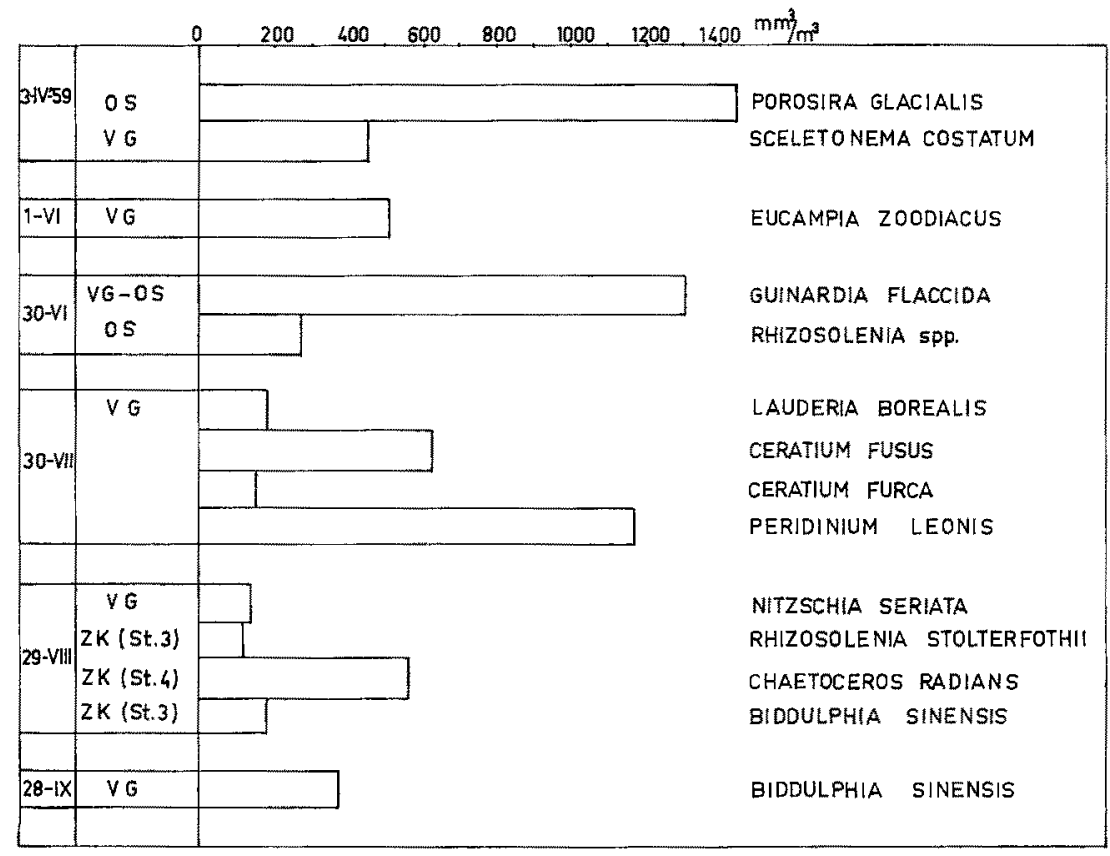

Abb. 5: Populationsvolumen der quantitativ wichtigsten Diatomeen und Dinoflagellaten im Veersche Gat und in der Zandkreek. Frühling und Sommer 1959

möglich, Mittelwerte für einen Jahreszyklus zu geben. Ganz allgemein aber läßt sich sagen, daß mit dem VG-Flutwasser Planktonorganismen in die ZK transportiert werden und daß die Planktondichte des Küstenwassers (VG) größer ist als die des ZKWassers. Mitunter erreichen einzelne Arten in der ZK höhere Dominanzien als im ursprünglichen VG-Wasser, welches sie herangeführt hat. Beim untersuchten Zyklus war das im August der Fall. Die absoluten Höhen der Frühlingsmaxima - immer durch Kieselalgen verursacht - können stark wechseln, wie aus unseren OS-Untersuchungen der letzten Jahre hervorgeht. Das Porosira-Maximum der OS im Jahre 1959 betrug etwa $1500 \mathrm{~mm}^{3} / \mathrm{m}^{3}$, während sich 1962 ein Frühlingsphytoplanktonvolumen von etwa $4000 \mathrm{~mm}^{3} / \mathrm{m}^{3}$ ergab. Im Vergleich mit (umgerechneten) Angaben anderer Autoren dürfen wir voraussetzen, daß Entwicklungsmaxima von etwa $3000 \mathrm{~mm} / \mathrm{m}^{3}$ gute Mit- 
telwerte für die europäische nordatlantische Küstenregion darstellen. In den obenstehenden Betrachtungen wurde ausschließlich vom Phytoplankton des Flutwassers gesprochen. Die Ebbwasserproben lassen wir hier außer Betracht, weil die geringfügigen Unterschiede sich nur auf einen relativ größeren Gehalt an littoralen Kieselalgen beziehen. Für quantitative Vergleiche bieten die Proben wenig Neues, und eine Erörterung kann daher unterbleiben.

$\mathrm{D}$ as Zooplankton. Innerhalb der Copepoden waren von quantitativer Bedeutung die Calanoida mit hauptsächlich den folgenden Arten: Temora longicornis, Acartia tonsa, Acartia clausi und Pseudocalanus minutus elongatus samt ihrer Nauplien- und Copepoditenstadien, ferner die Harpacticoida mit der pelagischen Euterpina acutifrons, im August und September der dominierende Copepodenvertreter, und littorale und benthonische Arten, die nicht qualitativ, sondern nur quantitativ bearbeitet wurden. Die Cyclopoida, mit Cyclopina spp. und anderen, sind littoral und erreichten niemals große Abundanz. Die Cirripedier waren mit den Nauplien und Cypris-Stadien von Balanus balanoides, Balanus crenatus und Elminius modestus zeitweilig lokal von großer quantitativer Bedeutung. Mit einigen Arten der Cladocera (aus den Gattungen Podon und Evadne), einigen Arten der Mysidacea (Schistomysis kervillei und Mesopodopsis slabberi) und Decapoden-Larven erreichten die planktonischen Crustacea eine Gesamtzahl von etwa 20 Arten, von denen aber weniger als 10 quantitativ wichtig waren. Von den weiteren Gruppen seien wegen ihrer quantitativen Bedeutung zuerst genannt die Larvenstadien der Lamellibranchiaten, Gastro-

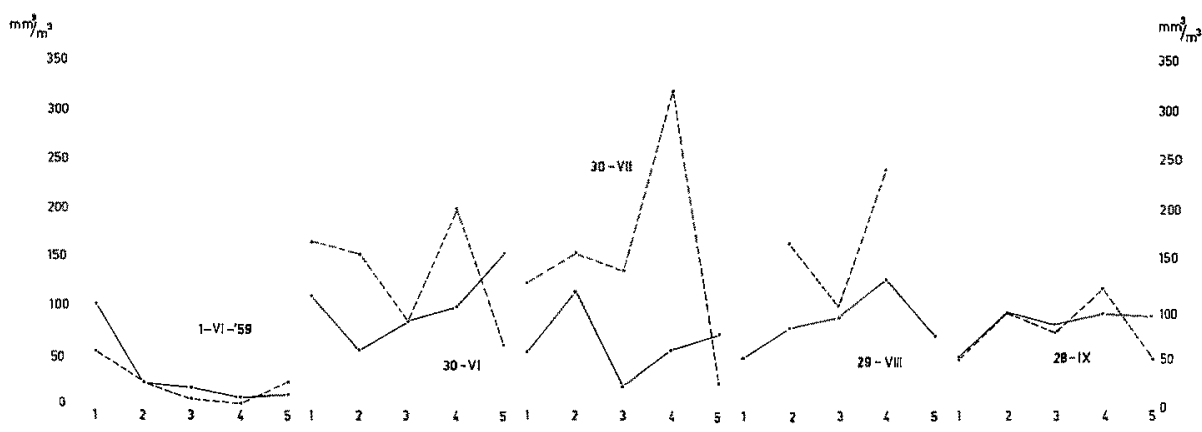

Abb. 6: Gesamtzooplanktonvolumen im Sommer 1959 des Veersche Gat und der Zandkreek $\left(\mathrm{mm}^{3} / \mathrm{m}^{8}\right)$. Kontinuierliche Linien: Flutwasser, unterbrochene Linien: Ebbewasser. Flut- und Ebbewasser einige Meter unterhalb der Oberfläche

poden und Polychaeten, die Appendicularien (mit zahlreichen Oikopleura dioica und vereinzelten Fritillaria borealis acuta) die Rotatorien (mit Trichocera marina marina und einigen mitunter abundanten Synchata-Arten, z. B. Synchata littoralis) und die Cystoflagellaten mit Noctiluca. Schließlich wurden regelmäßig beobachtet Chaetognathen (Sagitta setosa), Ciliaten (Arten der Oligotrichengattung Strombidium) und mehrere Arten der Tintinnidae, im Sommer außerdem Medusen und Larvenstadien von Echinodermen.

Vergleichen wir jetzt die Zooplanktonbiomassen der Stationen 1 bis 5, so ergibt sich die in Abbildung 6 wiedergegebene Situation. Man achte darauf, daß - im Gegen- 
satz zu der Darstellung in Abbildung 4 - die Kurven nicht für Flutwasserproben, welche der Oberfläche bzw. der Tiefe entnommen sind, gelten, sondern für Wasserproben aus einigen Metern unterhalb der Oberfläche, welche bei Ebbe und Flut entnommen worden sind. Die Unterschiede im Gesamtzooplanktonvolumen im Ebbe- und Flutwasser sind im Sommer sehr stark. So zeigen die Ebbeproben im ZK-Gebiet meistens höhere, für die Station 4 sogar erheblich höhere Planktonvolumen als die Flutproben. Die folgenden Zooplankter dominieren innerhalb der zentralen ZK im Sommer: Acartia tonsct, littoral-benthonische Harpacticiden, Cirripedier-Nauplien, Gastropoden und Synchaeta littoralis. Vor allem die Harpacticiden und die CirripedienNauplien zeigen deutliche Maximalwerte bei Station 4. Diese Gruppen bestimmen das Gesamtzooplanktonvolumen dieser "wantij"-Stelle so sehr, daß hier, gerade im Gegensatz zu den Verhältnissen des Phytoplanktons, die größten Biomassen gefunden werden. Der Seepockenaufwuchs der Landungsbrücken, Holzbekleidungen und Deiche erzeugt an Ort und Stelle zahlreiche Nauplien. Diese beeinflussen das Planktonbild erheblich, weil das Wasser dieser "wantij"-Station niemals ganz durch neues ersetzt wird, sondern mit den Gezeiten hin- und herpendelt. In den durch die Sommerkurven repräsentierten Proben aus halber Tiefe wird der Einfluß des Ebbestroms deutlich sichtbar: Die autochthonen Nauplien der Harpacticiden und Balaniden des "Wantij"-Gebiets befinden sich in den unteren Wasserschichten. Sie werden daher durch die Ebbe leicht aufgewirbelt und erscheinen dann massenhaft in den Proben. Auch Flutproben enthalten autochthones Material, aber in viel geringeren Mengen (Abb. 6). Für mehrere Arten ist dieselbe Situation nachzuweisen wie für die Phytoplanktonproben, nämlich Transport aus dem VG.

\section{UNTERSUCHUNGEN IM ABGESCHLOSSENEN MEERESARM, VEERSE MEER, 1961 BIS HEUTE}

Die wichtigsten physikochemischen Veränderungen nach Abtrennung des Meeresarms lassen sich wie folgt charakterisieren: 1. Ausfallen der Gezeiten; 2. Abnahme des Salzgehalts; 3. Eutrophierung; 4. Zufrieren während strenger Winter. Die biologischen Folgen dieser Änderungen lassen sich meistens nicht im einzelnen auf einen bestimmten Faktor, wie etwa die Herabsetzung des Salzgehalts oder zunehmende Eutrophierung zurückführen. Vor allem haben Veränderungen der Faktoren 2 und 3 eine Reaktionskette von weiteren Anderungen eingeleitet. Das erschwert natürlich eine genaue kausalanalytische Beurteilung der biologischen Konsequenzen der Abschließung des Meerbusens. Nur von einer Kombination einer Deskription der aktuellen Anderungen und einer experimentellen Prüfung der herabgesetzten Chlorinität sowie des gesteigerten Nährstoffgehalts kann man sich befriedigende Schlußfolgerungen erhoffen. Zur Zeit befinden wir uns noch in der ersten Untersuchungsphase, aber die Vorbereitungen zur zweiten Phase haben begonnen.

\section{Ausfallen der Gezeiten}

Die Anwesenheit des westlichen Dammes schließt jede unmittelbare Zufuhr von Organismen aus der Nordsee ins Veerse Meer (VM) aus. Wir zeigten schon die wesent- 
liche Rolle der VG-Zufuhr für die quantitativen und qualitativen Aspekte der planktonischen Besiedelung der ZK. Mehrere Arten waren nur dank des Transportes aus dem VG nachzuweisen: Im Zooplankton z. B. die rein planktonischen, nicht streng neritischen Arten, welche keine littoral-benthonischen Stadien aufweisen, wie etwa die Copepoden Labidocera wollastoni und Calanus finmarchicus und die Appendicularie Fritillaria borealis acuta. Auch vielen anderen Arten, z. B. Larvenstadien verschiedener Fische, dem Chaetognath Sagitta setosa, sowie Hydro- und Scyphomedusen wurde plötzlich der Zutritt aus dem Westen unmöglich gemacht. Diese Arten wurden daher sogleich nach der Abdeichung ausgeschaltet. Dieser rein-mechanische Effekt spielte gleichfalls eine wichtige Rolle bei der Abschließung der Zuiderzee im Jahre 1932, jetzt Ijsselmeer (vgl. DE BEAUfORT 1954).

Gelegentliche Zufuhr von OS-Wasser ist aber noch immer möglich. Der östliche Damm ist nämlich mit Schleusen versehen. Während des ersten Jahres nach der endgültigen Abdeichung, 1961, war diese gelegentliche OS-Zufuhr sicher wichtig. Die Umweltsituation im VM hatte sich noch nicht so eingehend geändert, jedenfalls nicht in bezug auf den Chloridgehalt, daß marine Arten aus der OS im VM nicht hätten gedeihen können. Alljährlich wird im Frühling der Wasserstand im VM etwa $70 \mathrm{~cm}$ erhöht durch Zuführung von OS-Wasser und im Herbst dann eine entsprechende Menge wieder in die OS abgeführt. Es gibt also Möglichkeiten für eine regelmäßige Zufuhr von Organismen ins VM. Allerdings ist der Effekt der Wasserzufuhr schon wenige Kilometer westwärts an Hand von Planktonproben nicht mehr nachweisbar. Mit zunehmender Veränderung der Umweltbedingungen im VM nimmt der Einfluß der OS-Zufuhr immer mehr ab und ist schon heute für die Besiedlung dieses salzigen Binnengewässers nur noch von nebensächlicher Bedeutung.

Eine zweite Folge des Ausfallens der Gezeiten war das Auftreten von Planktonschichtungen. Der nivellierende Effekt des turbulenten Flut- und Ebbewassers erlaubte niemals die Entstehung einer Stratifikation. Nur Sedimentationsphänomene (Herabsinken der Planktonorganismen und des Triptons bei Stillwasser) traten im Gezeitengebiet auf. Allerdings wurden deutliche Temperatur- und Salzgehaltsschichtungen vorerst (bis Anfang 1963) noch nicht beobachtet. Wegen der geringen mittleren Tiefe des VM ist der Effekt des Windes groß, und so wird eine im Entstehen begriffene Stratifikation bald wieder aufgehoben. Auch die tieferen Areale $(20-25 \mathrm{~m})$ weisen keine Schichtung auf.

\section{Herabsetzung des Salzgebalts infolge von Niederscblagsüberschüssen und abgefübrten Polderwassers}

Nachdem der östliche Damm im Frühling 1960 fertiggeworden war, ließ sich während des Sommerhalbjahres noch kein Einfluß dieser Abtrennung auf den Salzgehalt nachweisen. Im Herbst aber sank die Chlorinität zeitweilig bis auf $10 \% \mathrm{Cl}$ an der äußersten Ostseite ab. Im Gegensatz dazu blieb die Chlorinität an der Westseite der VG bei normalen Ebbe- und Flutbedingungen auf 16-17\% $\mathrm{Cl}$ stehen. Als im April 1961 der VG-Damm zustande kam, wurde das VM auf der ganzen Strecke fast gleichförmig in bezug auf die Chlorinität mit einem Mittelwert von etwa 16,5\% Cl. Diese 
Situation dauerte bis Oktober 1961. Dann begann ein kontinuierliches Absinken. Ende November waren ungefähr 13,5\% Cl erreicht, Mitte Januar 1962 10-11\% $\mathrm{Cl}$, und Anfang März 1962 fielen die Werte sogar unter 10\% Cl ab. Im Laufe des Sommers 1962 stieg die Chlorinität dann wieder allmählich bis über $11 \% \mathrm{Cl}$ an, ohne daß es dabei zu einer Salzgehaltsschichtung gekommen wäre. Abgesehen von geringfügigen Unterschieden zwischen Oberflächen- und Tiefenwasser und kleinen Differenzen zwischen den verschiedenen Proben im Horizontalprofil erwies sich der Salzgehalt als homogen im ganzen Bereich des VM. Diese Situation hat sich in bezug auf das Oberflächenwasser während des Jahres 1963 wiederholt. Zusammenfassend ergibt sich, daß das heutige VM augenblicklich ein meso-polyhaliner Biotop geworden ist. Vorläufig ist anscheinend ein gewisses Gleichgewicht erreicht. Für eine endgültige, progressive Aussüßung ist selbstverständlich die erst im Jahre 1978 geplante Abdeichung der OS von ausschlaggebender Bedeutung.

Eine der eindrucksvollsten Wirkungen der Herabsetzung der Chlorinität ist das Verschwinden vieler euhalin-polyhaliner Arten, vor allem vieler Diatomeen und Copepoden - zweier wichtiger Gruppen des Planktons im ehemaligen VG-ZK-Bereich. Nahezu alle euhalin-polyhalinen Kieselalgen sind aus dem VM verschwunden. Während des Sommers 1961, als der Salzgehalt ja noch etwa 16,5\%00 $\mathrm{Cl}$ betrug, waren noch mehrere Diatomeen aus der ehemaligen Lebensgemeinschaft vorhanden: Verschiedene Chaetoceros-Arten, einige Rbizosolenia-Arten, Leptocylindrus danicus, Bacteriastrum byalinum und andere. Damals aber schon blieb diese Anzahl weit hinter derjenigen der OS zurück. Im Frühling 1962 waren an der Massenentwicklung beteiligt die Arten Lauderia borealis, Thalassiosira nordenskioeldi und Sceletonema costatum. Die Sommerproben enthielten noch Sceletonema, und im Frühling 1963 war Sceletonema die einzige Komponente der ursprünglichen planktonischen Diatomeengemeinschaft. Hinsichtlich der Copepoden ist zu bemerken, daß nur noch eine Art der ursprünglichen Küstenvertreter sich halten konnte: Acartia tonsa. Im Sommer 1963 zeigte sich Temora longicornis ziemlich regelmäßig im östlichen Teil des VM, während diese Art im vorhergehenden Jahr nur vereinzelt gefunden wurde. Das gelegentliche Wiederauftreten dieser Art ist als ein Beispiel der Zufuhr von OS-Wasser durch die Katseveer-Schleusen zu betrachten.

Gleichzeitig mit der Ausschaltung mariner Arten wurde eine maximale Abundanz der übrigbleibenden Arten beobachtet. Sceletonema costatum erreichte Maxima von etwa $100 \times 10^{6}$ Zellen/1 (Mittelwert für eine Wassersäule von $10 \mathrm{~m}$ ), während diese Art zur gleichen Zeit in der OS maximal "nur" $5-15 \times 10^{6}$ Zellen/ 1 aufwies (gleichfalls Mittelwerte für eine Wassersäule von $10 \mathrm{~m}$ bei Hochwasser). Die vorstehenden Werte aus der OS sind höher als die von LoHMAnN (1908) in der Kieler Bucht für Entwicklungsmaxima von Sceletonema gefundenen, aber niedriger als die von MarSHALl \& ORR (1927) gleichfalls für Sceletonema erhaltenen Werte im Clyde Sea Bereich. Acartia tonsa erreicht im Sommer im VM in den tieferen Wasserschichten Konzentrationen von 500-6000 Exemplaren/100 1. In der ehemaligen ZK wurde eine Dichte nachgewiesen von nur 200-600 Exemplaren/100 1, gleichfalls in den tieferen Wasserschichten bei Stillwasser. Enorme Mengen Polychaetenlarven der Gattung Polydora verwandelten im Mai 1963 das VM-Wasser in eine "Polychaeten-Suppe“: 8000 bis 20000 Exemplare/100 l (wieder Mittelwerte eines Vertikalprofils von etwa $10 \mathrm{~m}$ ). 
Polydora-Larven erreichten in der ZK (und erreichen in der OS) niemals höhere Werte als 500 Exemplare/100 1 (Mittelwert für eine $10 \mathrm{~m}$ tiefe Wassersäule bei Stillwasser). Übrigens ist die größere Planktondichte nicht ohne weiteres auf die geringere Konkurrenz zwischen den wenigeren Arten eines brackigen Biotops zurückzuführen, sondern auch auf die zunehmende Eutrophierung.

\section{Die Eutrophierung des Veerse Meer infolge abgefübrten Polder- und Abwassers}

Während des ersten Jahres nach der endgültigen Abtrennung (1961-1962) wurde das Abwasser der Stadt Middelburg (Abb. 1) noch immer in das VM bei Veere abgeführt: $3000 \mathrm{~m}^{3}$ pro Tag, ungereinigt. (Zugleich mit der Abdeichung des VG war eine Abwasserabfuhr auf die Westerschelde geplant, aber diese kam erst ein Jahr später zustande.) Kombiniert mit den großen Mengen Polderwasser führte Vorstehendes schon im Sommer 1961 zu einer starken Anreicherung des Nährstoffgehaltes des Wassers, die unter anderem in hohen Phosphat-Zahlen zutage trat. Statt etwa $5 \mu$ gr.at.P/1 wurden jetzt Werte von $20-40 \mu$ gr. at. P/1 erreicht. Diese hohen Gesamtphosphorwerte waren hauptsächlich dem $\mathrm{PO}_{4}$-Ion und gelöstem organischen Phosphor zuzuschreiben.

Die deutliche Folge der zunehmenden Eutrophierung war das erstmalige Auftreten mehrerer brackig-saprober Arten. Neben der drastischen Verringerung der Anzahl von Diatomeenarten fällt ferner der große Anteil auf, den sich die Flagellaten im Nannoplankton des VM eroberten. Als Beispiel seien genannt Rhodomonas pelagica sowie Chlamydomonas- und Pyramimonas-Arten. In dem Maße, in dem Sceletonema allmählich aus dem Planktonbild verschwindet, treten Dinoflagellaten auf, z. B. Glenodinium lenticula f. minor, Massartia rotundata, Gonyaulax spinifera, Peridinium triquetrum, Gymnodinium spp. Mehrere dieser Flagellatenarten wurden auch in der ehemaligen Zuiderzee beobachtet (REDEKE 1922, 1936). Im marinen Gezeitengebiet der OS beträgt die Gesamtmenge der Flagellaten 4000-6000 Zellen/cc (DrunKWAARD 1958), aber im VM wurden Maxima von 20-50 $\times 10^{3}$ Zellen/cc gezählt. Auffallend war die langdauernde Dominanz einer Gymnodinium-Art im Sommerplankton von 1963. Weil gleichfalls eine üppige Bakterienflora im eutrophierten Wasser wächst, können verschiedene Protozoa im VM vorzüglich gedeihen. Im Frühling dominieren der Kieselflagellat Ebria tripartita (als Sceletonema-Konsument; Maximum 2200 Exemplare/cc) und die Ciliaten Euplotes harpa (210 Exemplare/cc) und Wesodinium pulex (230 Exemplare/cc).

Der Einfluß der Eutrophierung war im Sommer 1961, als der Salzgehalt noch nicht herabgesetzt war, schon nachzuweisen durch ein Entwicklungsmaximum von Prorocentrum spec. (nicht die bekannte Art P. micans) im VM. Dieser Organismus stammt aus der Nordsee und wurde in den Mündungen der OS und Westerschelde nachgewiesen. Aus Untersuchungen des Fischereilaboratoriums in Ijmuiden über eine zu derselben Zeit aufgetretene Muschelvergiftung ging hervor, daß im Darmkanal der vergifteten Muscheln große Mengen von Prorocentrum spec. aufzufinden waren. Die Abundanz in der OS war nur gering: maximal einige Hunderte von Exemplaren/cc. Im VM, welches regelmäßig zusammen mit dem Middelburger Abwasser aus der 
Westerschelde durch den Kanal von Walcheren Verdünnungswasser bezog (Abb. 1), entwickelte die Art sich rasch, und innerhalb von 2 Wochen wurden Werte von 1-10 $\times 10^{3} \mathrm{Zellen} / \mathrm{cc}$ festgestellt. Dieses markante Entwicklungsmaximum wurde z weifelsohne durch die verunreinigten VM-Wässer gefördert.

Aus den obigen Beispielen und den erwähnten Massenentwicklungen - insbesondere von Sceletonema und Polydora-Larven - geht hervor, daß die herabgesetzte Chlorinität und der gesteigerte Trophiegrad zusammenwirken. Einerseits wird die normale Konkurrenz gemildert, weil viele Arten durch den verringerten Salzgehalt ausgeschaltet worden sind, andererseits ist eine außerordentlich günstige Umwelt für andere (saprobe) Arten entstanden. Für Sceletonema, als eine leicht saprobe und stark euryhaline Art, addieren sich die positiven Wirkungen beider Faktoren und führen zu sehr großen Biomassen. Uber die basalen Glieder der Nahrungskette (Bakterien, Phytoflagellaten, farblose Flagellaten, Kieselalgen) wird dann auch eine Massenentwicklung der folgenden Stufen in der Nahrungspyramide ausgelöst: Protozoen, Wurmlarven, Copepoden, Mysideen, Fische.

Es ist uns an dieser Stelle nicht möglich, eine detaillierte Übersicht über die jahreszeitlichen Fluktuationen der Planktonvolumina des VM zu geben. Ich beschränke mich auf die Angabe einiger Zahlen. Diese deuten auf sehr unregelmäßige Schwankungen hin, besonders hinsichtlich der Horizontalprofile und der zeitlichen Aufeinanderfolge der Planktongemeinschaften. Die Schwankungen sind charakteristisch für die unstabilen Verhältnisse im VM, die sich wohl auch in nächster Zukunft noch erhalten werden. Ein Vergleich der Biomassen des ehemaligen VG-ZK-Wassers und denen des heutigen VM während verschiedener Jahreszeiten zeigt, daß die letzteren immer wesentlich größer sind. Maxima von $20 \times 10^{3} \mathrm{~mm}^{3} / \mathrm{m}^{3}$ Nannoplankton sind für das VM durchaus üblich, ja, diese Werte werden im Frühling und im Sommer noch erheblich überschritten. Ein Beispiel für sehr hohe Zooplanktonvolumina liefern die Netzproben vom Mai 1963 mit dem erwähnten Maximum der Polydora-Larven mit etwa $6000 \mathrm{~mm}^{3} / \mathrm{m}^{3}$. Die Nannoplankton-Biomasse sank demnach in diesem Monat bis auf etwa 8500 $\mathrm{mm}^{3} / \mathrm{m}^{3}$ nach einem Frühlingsmaximum Mitte April von $\pm 30 \times 10^{3} \mathrm{~mm}^{3} / \mathrm{m}^{3}$.

Eine deutliche Vergrößerung der Biomasse wies auch der Fischbestand auf. Besonders für die Bodenfische liegen viele Angaben vor. Nach Beobachtungen von Dr. K. F. VAAs (Yerseke) bildete die Scholle (Pleuronectes platessa L.) nach der Abdeichung den größten Teil der Gesamtbiomasse der Fische und wachs kontinuierlich an Individualgewicht. Ahnliches gilt für den Aal, Anguilla anguilla (L.), und die Aalquappe, Zoarces viviparus (L.).

Infolge der starken Entwickłung des Nannoplanktons in den oberflächlichen Wasserschichten nimmt die Durchsichtigkeit des Wassers ab. So schwanken die Ablesungen mit der Secchi-Scheibe um $70 \mathrm{~cm}$ Sichttiefe im Frühling und im Vorsommer. In dieser Zeit liegt der Kompensationspunkt in etwa $3 \mathrm{~m}$ Tiefe (nach Dr. K. F. VAas). Unterhalb einer Tiefe von $10 \mathrm{~m}$ kann sich demnach der Sauerstoffgehalt während warmer und windstiller Tage rasch senken. Im Sommer 1962 hatte dieser Umstand ein verhängnisvolles Fischsterben zur Folge, welches nicht nur die Bodenfische Pleuronectes platessa L., P. flesus L. und Cottus scorpius L. betraf, sondern auch pelagische Arten, wie Clupea barengus L., sowie Gobius minutus Paldas, einen Bewohner der seichten Küstengewässer. $A b$ und $z u$ waren an tieferen Stellen schon früher tote Bodenfische 
heraufgeholt worden. Der Sauerstoffschwund hatte auf das Plankton geringen Einfluß. Nur die vertikale Zooplanktonschichtung war gestört, weil die erwachsenen Acartien sich tagsüber an der Oberfläche befanden, und zwar in derart großer Zahl, daß auf dem Schlammboden in sehr seichtem Wasser viele Exemplare in Schöpfproben gezählt wurden.

Die Entstehung des brackig-eutrophen Biotops verursachte bei einigen Arten Wuchsanomalien. Als Beispiel sei die Wuchsform von Sceletonema costatum im Frühling 1963 erwähnt. Die Kolonien bestanden hauptsächlich aus paarweise zusammenhängenden Zellen oder 4zelligen Fäden. Langgestreckte Kolonien waren selten. Die Kieselstäbchen, welche die Verbindung zwischen zwei Zellen bilden, waren nur schwach entwickelt und oft gar unsichtbar. Der veränderte Habitus von Sceletonema ist zu erklären, wenn wir ein stark beschleunigtes Wachstum infolge der saproben Umwelt annehmen. Möglicherweise vermag die Bildung der Kieselstrukturen nicht mit der gesteigerten Tellungsfrequenz Schritt zu halten. Ferner dürfte die große Zartheit der Kieselstäbchen die Bildung langer, zusammenhängender Individuenketten ausgeschlossen haben und eine Kette nach ein oder zwei Teilungen zerfallen sein.

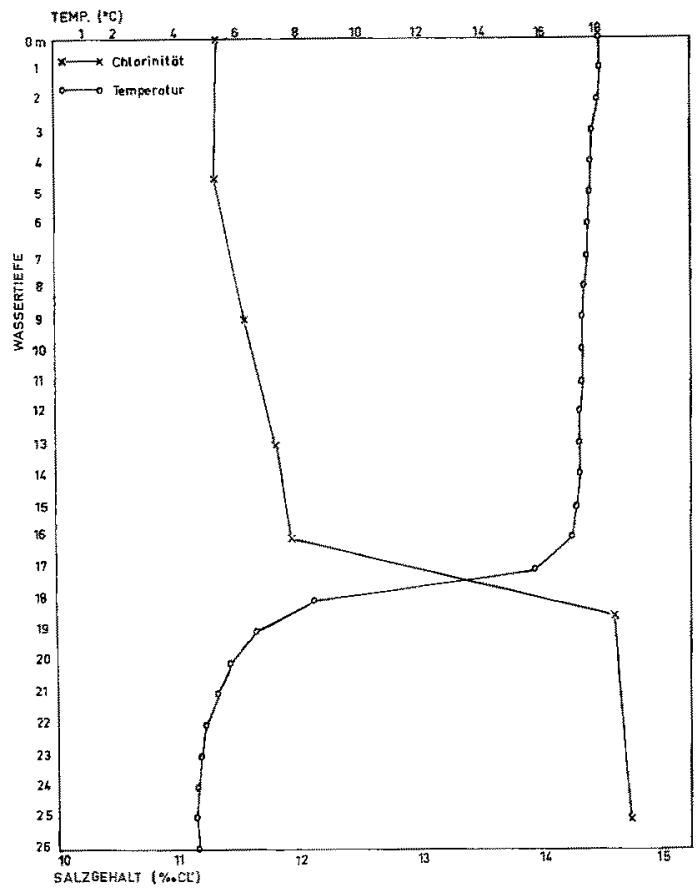

Abb. 7: Temperatur- und Chlorid-Vertikalprofile einer tiefen Stelle des Veerse Meer am 12. Juli 1963. Station VM 21. Doppelschichtung in einer Tiefe von $16-19 \mathrm{~m}$

Totales Zufrieren des Veerse Meer wäbrend des kalten Winters 1962/63

Deutliche Salzgehalts- oder Temperaturschichtungen wurden bis Januar 1963 nicht beobachtet. Schon erwähnt wurde der große Windeinfluß auf das im allgemeinen flache 
VM, welcher Vertikalschichtungen im allgemeinen verhinderte. Erst die strenge Kälte Anfang 1963 änderte diese Situation. Während der letzten Monate des Jahres 1962 schwankte der Salzgehalt um $12 \% \mathrm{Cl}$ herum mit Differenzen zwischen Oberflächenund Tiefenwasser von etwa $0,5 \% \mathrm{Cl}$. In der gut 2 Monate dauernden Frostperiode sank die Chlorinität des Oberflächenwassers ein wenig (bis auf 11,5\%); gleichzeitig

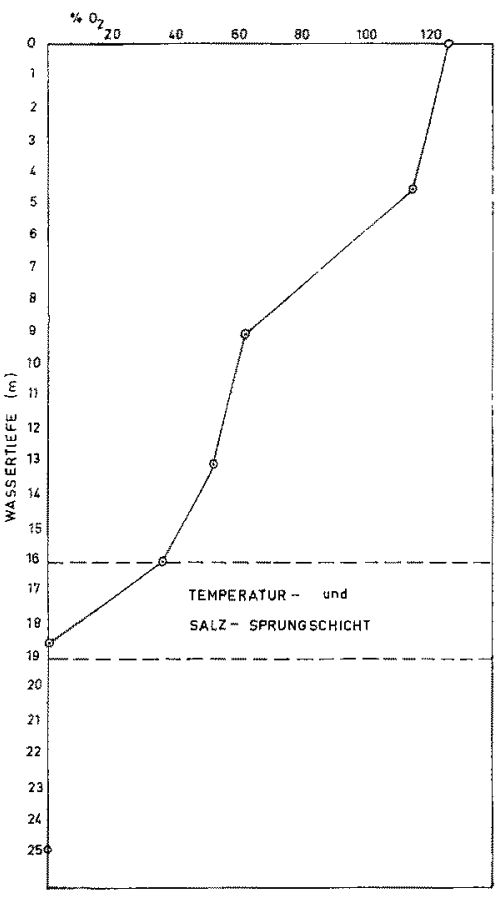

Abb. 8

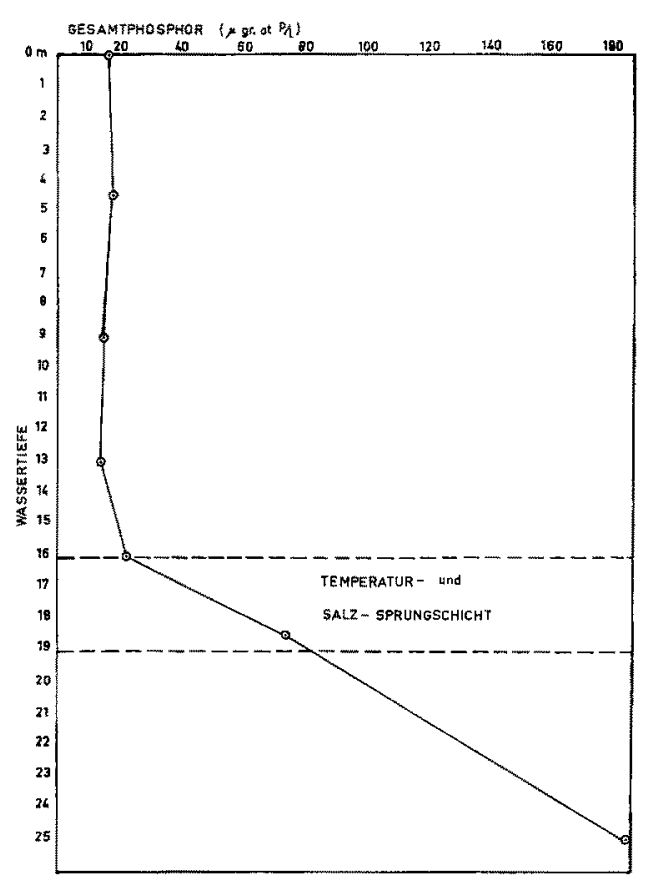

Abb. 9

Abb. 8: Verringerung der Sauerstoffsättigungswerte im Veerse Meer bei der Station VM 21 (12. Juli 1963)

Abb. 9: Steigerung des Gesamtphosphors im Veerse Meer auf der Station VM 21 (12. Juli 1963)

stieg die Chlorinität in $10 \mathrm{~m}$. Tiefe und darunter auf 14 bis $15 \% \mathrm{Cl}$ an. Die in dieser Weise entstandene Salzgehaltsschichtung wurde nach dem Eintritt des Tauwetters nicht zerstört und wurde bald von einer Temperaturschichtung begleitet (Abb. 7). Durch diese für brackige Gewässer so charakteristische Kombination der Temperatur- und Salzgehaltsstratifikation (REMANE 1958) wurde die Stabilität dieser Doppelschichtung so groß, daß diese trotz der geringen Tiefe des VM bis August 1963 bestehen blieb. Allerdings wurde die Sprungschicht durch starke Mischung des Oberflächenwassers hinabgedrückt, so daß sie im Laufe des Sommers nur noch in den tiefsten Teilen als dünne Bodenschicht aufzufinden war. Schließlich verschwand sie auch hier.

Auch die Begleiterscheinungen einer stabilen Schichtung im Brackwasser, wie Sauerstoffschwund (Abb. 8) und Schwefelwasserstoffreichtum unterhalb der Sprungschicht, wurden beobachtet. Ferner werden die Verhältnisse in Binnengewässern be- 
kanntlich noch dadurch kompliziert, daß unterhalb der Sprungschicht der Ionenaustausch zwischen Schlamm und Bodenwasser zu einer großen Anreicherung von Nährstoffen führt. Zwei Beispiele: Der Gehalt an Phosphor ( $\mu \mathrm{g} \cdot$ at $\cdot \mathrm{P} / 1$; Abb. 9) nahm von der Sprungschicht an nach unten hin sehr stark zu, und zwar von etwa 20 auf $185 \mu \mathrm{g}$. at $\cdot \mathrm{P} / 1$. Der größte Teil dieses Anstiegs (130 $\mu \mathrm{g} \cdot$ at $\cdot \mathrm{P} / \mathrm{l})$ geht auf die Rechnung des $\mathrm{PO}_{4}$-Ions. Ahnliches galt für das Silicium: Der Si-Gehalt des Oberflächenwassers betrug $225 \mu \mathrm{g} \mathrm{Si} / \mathrm{l}$, der des Tiefenwassers aber $4400 \mu \mathrm{g} \mathrm{Si} / 1^{3}$.

\section{ZUSAMMENFASSUNG}

Es wird eine kurze Übersicht über Umweltfaktoren und Plankton eines ehemaligen Meerbusens vor der Abdeichung (Veersche Gat-Zandkreek) und nachher (Veerse Meer) gegeben. Für die quantitave Erfassung des Planktons wurde die Methode von LOHMANN (1908) angewandt.

In bezug auf das Veersche Gat und die Zandkreek sind die wichtigsten Ergebnisse:

1. Als Teil des Oosterschelde-Systems repräsentierten Veersche Gat und Zandkreek ursprünglich euhalin-polyhaline Biotope. Durch ein "wantij" bei Wolfaartsdijk wurde eine partielle Isolation der zentralen Zandkreek verursacht mit dem Ergebnis, daß abweichende Chlorinitätswerte und signifikante Veränderungen in der qualitativen und quantitativen Zusammensetzung der Planktongemeinschaften auftraten.

2. Das Zandkreek-Plankton bestand aus einer neritischen Lebensgemeinschaft, wobei sich das Phytoplankton vornehmlich aus pelagischen Kieselalgen zusammensetzte, das Zooplankton hingegen stark litorale Züge aufwies.

3. Die Biomasse nahm im allgemeinen seewärts zu.

4. Das Phytoplankton im "wantij“ zeigte zum Teil autochthone Entwicklungstendenzen, zum Teil auch starke Massenentwicklung.

5. Infolge einer starken autochthonen Entwicklung litoraler Zooplanktonarten innerhalb der zentralen Zandkreek im Sommer waren hier größere Biomassen nachzuweisen als im Veersche Gat.

Im Hinblick auf das Veerse Meer ergab sich:

1. Die Herabsetzung des Salzgehalts im neu entstandenen Veerse Meer hat während der letzten zwei Jahre zu der Bildung eines $\alpha$-meso-polyhalinen Biotops geführt.

2. Die partielle Aussüßung wurde begleitet von einer starken Eutrophierung des Wassers, welche unter anderem in hohen Phosphorgehalten zutage trat.

3. Die Ausschaltung vieler polyhalin-marinen Arten wurde durch das Auftreten vieler Brackwasserarten kompensiert, unter denen Flagallaten und Dinoflagellaten vorherrschen.

4. Die heutige Besiedelung ist gekennzeichnet durch Biomassen, die um das Mehrfache größer sind als diejenigen der Zandkreek und des Veersche Gat.

${ }^{3}$ Für die Bestimmung danke ich Herrn Dr. H. L. Golterman, Hydrobiologisch Instituut, Nieuwersluis. 
5. Der strenge Winter 1962/63 hatte eine ausgeprägte Salzgehaltsschichtung zufolge, die zusammen mit einer Temperaturstratifikation als Doppeltschichtung bis August 1963 nachweisbar war.

Id danke den Herren Drs. A. M. P. KaAshoek (Goes) und Prof. Dr. O. Kinne (Hamburg) für das Korrigieren des deutschen Textes.

\section{ZITIERTE LITERAT'UR}

BEAuforT, L. F. DE, C. S., 1954. Changes in the flora and the fauna of the Zuiderzee (now Ijsselmeer) after the closure in 1932. Verlag C. de Boer Jr., Den Helder, 359 pp.

DRINKWAARD, A. C., 1960. The quality of oysters in relation to environmental conditions in the Oosterschelde in 1958. Annls biol, Copenh. 15, 224-233.

Hartog, C. Den, 1961. Die faunistische Gliederung im süd-west-niederländischen Deltagebiet. Int. Rev. Hydrobiol. 46, 407-418.

Hoekman, J. M., 1962. Onderzock van watermonsters van de Zandkreek vóór en ná de afsluiting. (Interner Bericht des Hydrobiol. Instituts, Yerseke; nicht publiziert.)

Lohmann, H., 1908. Untersuchungen zur Feststellung des vollständigen Gehalts des Meeres an Plankton. Wiss. Meeresunters. Kiel 10, 129-370.

Marshall, S. M. \& ORR, A. P., 1927. The relation of plankton to some chemical and physical factors in the Clyde Sea Area. J. Mar. biol. Ass. U. K. 14, 837-868.

Renexe, H. C., C. S., 1922. Flora en Fauna der Zuiderzee. Monografie van een Brakwatergebied. C. de Boer Jr., Den Helder, 460 pp.

- 1936. Idem. Supplement. 176 pp.

Remane, A. \& Schlieper, C., 1958. Die Biologie des Brackwassers. In: Die Binnengewässer. Bd 22, Schweizerbart, Stuttgart.

Schudrmans Stekhoven, J. H., 1961. An investigation of the planktonic population of diatoms of the "Zandkreek" during the period March 1959 to March 1960. Act. bot. neerl. 10, 320-326.

VAAS, K. F., 1961. Das Arbeitsfeld des Hydrobiologischen Institutes, Abteilung Deltauntersuchungen, in den Niederlanden. Int. Rev. Hydrobiol. 46, 292-295. 